\title{
A Novel Stackelberg-Bertrand Game Model for Pricing Content Provider
}

\author{
Cheng Zhang \\ Department of Computer \\ Science and Communications \\ Engineering \\ Waseda University, Tokyo, \\ 169-8555 Japan \\ cheng.zhang@akane. \\ waseda.jp
}

\author{
$\mathrm{Bo} \mathrm{Gu}$ \\ Department of \\ Communications and \\ Computer Engineering \\ Waseda University, Tokyo, \\ 169-8555 Japan
}

\author{
Kyoko Yamori \\ Department of Management \\ Information \\ Asahi University, Gifu, \\ 501-0296 Japan
}

\author{
Sugang Xu \\ Global Information and \\ Telecommunication Institute \\ Waseda University, Tokyo, \\ 169-0051 Japan
}

\author{
Yoshiaki Tanaka \\ Department of \\ Communications and \\ Computer Engineering \\ Waseda University, Tokyo, \\ 169-8555 Japan
}

\begin{abstract}
With the popularity of smart devices such as smartphones, tablets, contents that traditionally be viewed on a personal computer, can also be viewed on these smart devices. The demand for contents thus is increasing year by year, which makes the content providers (CPs) get high revenue from either users' subscription or advertisement. On the other hand, Internet service providers (ISPs), who keep investing in the network technology or capacity to support the huge traffic generated by contents, do not benefit directly from the content traffic. One choice for ISPs is to charge CPs to share the revenue from the huge content traffic. Then ISPs will have enough incentives to invest in network infrastructure to improve quality of services (QoS), which eventually benefit CPs and users. This paper presents a novel economic model called Stackelberg-Bertrand game to capture the interaction and competitions among ISPs, CPs and users when ISPs charge CPs. A generic user demand function is assumed to capture the sensitivity of demand to prices of ISPs and CPs. The numerical results show that the price elasticity of ISP and CP plays an important part on the payoff of the ISP and CP.
\end{abstract}

\section{Categories and Subject Descriptors}

C.2.3 [Computer Communication Networks]: Network Operations-Network Management

\section{General Terms}

Economics, Management

\section{Keywords}

Network neutrality, Content provider, Internet service provider, Stackelberg-Bertrand game

\section{INTRODUCTION}

The Internet service providers (ISPs) keep investing in the network technology or capacity to provide better quality of service (QoS) for end users. However, new broadband applications generate huge amount of traffic, which originates from the existence of content providers (CPs), without generating direct revenues for ISPs. Hence, ISPs want to get additional revenues from CPs that are not directly connected to them. One choice for ISPs is to charge the CPs. Actually, whether ISPs can charge CPs or not remains the centre of network neutrality ${ }^{1}$ debate $[2][11][16]$.

In Japan, the Ministry of Internal Affairs and Communictions (MIC) has published a series of white papers related to network neutrality debate [1]. According to MIC, the pricing between ISPs and CPs can be left to consultation among ISPs and CPs. In this paper, non-network neutrality is assumed: the ISP can charge $\mathrm{CP}$ to cover the investment cost.

Ma et al. in [9][10] proposed Shapley value methods [12][14], which developed from cooperative game theory, to solve the revenue sharing problem between ISPs and CPs.

\footnotetext{
${ }^{1}$ network neutrality is the principle that Internet service providers and governments should treat all data on the Internet equally, not discriminating or charging differentially by user, content, site, platform, application, type of attached equipment, and modes of communication.
} 
Different from [9][10], in this paper, non-cooperative nature of ISPs and CPs is considered while ISPs and CPs compete with each other to maximize their revenue. Hande et al. in [6] generalized the well-known network utility maximization (NUM) based rate allocation model [8][4] to study the pricing of CPs under monopolistic and perfectly competitive ISPs markets. However, the NUM model highly depends on utility function, which can hardly be known exactly. In NUM model, the dual gradient mechanism developed from convex optimization theory [3] is always proposed to converges to the equilibrium prices when utility function is concave. In this work, generic demand function is adopted without any concavity assumption on the utility function. Recently, Joe-Wong et al. in [7] studied the impact of sponsored data plan by ISPs on users and CPs. A sponsored data plan provided by ISPs allows CPs to determine how much they pay for the network connectivity for network users. They shown that sponsored data plan benefit users proportionally more than CPs. However, CPs may choose do not use sponsored data plan provided by ISP, then ISPs investment cost may not be recovered by this way. Further more, competition is also omitted in [7].

In this paper, a simplified market, which is composed of one ISP, one CP, and large number of users is considered. The ISP charges end users based on their usage. The CP charges end users the subscription fee. Furthermore, the ISP also charges the CP to cover the investment cost. Please refer to Figure 1 for the money flow between the ISP, the CP and end users.

A Stackelberg leader-follower game [5] is a strategic game in which a leader player commits a strategy first and then other followers move sequentially. In the Bertrand game [15], different firms strategically choose prices independently at the same time while supplying quantities demanded at the chosen prices. Based on Stackelberg game and Bertrand game, a novel Stackelberg-Bertrand game is proposed to obtain the equilibrium price of ISP and CP. At the firststage of the Stackelberg-Bertrand game, the ISP sets the price for CP. Then in the second stage of the game, the ISP and the CP play a Bertrand game. We solved the equilibrium of the Stackelberg-Bertrand game by backward induction [5]. Given the price set for CP by ISP, the Bertrand game between ISP and CP is solved firstly. Then the ISP sets the optimal price to charge $\mathrm{CP}$ through backward induction.

The main contribution of this paper are as follows:

- A novel Stackelberg-Bertrand game is proposed to model the noncooperative behaviour between ISP and CP. Unique Nash equilibrium of the game is established.

- Generic user demand function is utilized to calculate the utility of ISP and CP.

- The effect of payment from CP to ISP is evaluated under different user demand functions.
The rest of this paper is organized as follows. Basic modle of ISP, CP and users are presented in Section 2. In section 3, a novel Stackelberg-Bertrand game modelling firstly, then unique Nash equilibrium of the game is established. Numerical results are presented in Section 4. Section 5 concludes this paper.

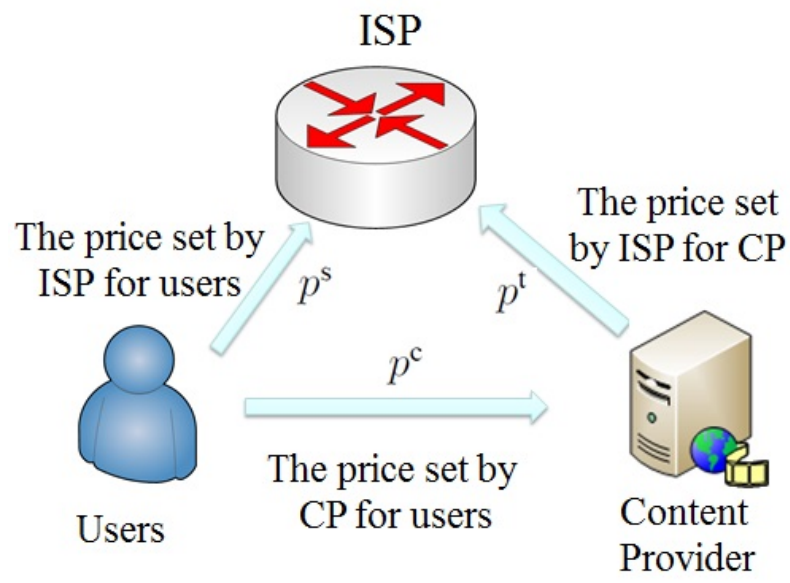

Figure 1: Money flow between ISP, CP and users.

\section{BASIC MODEL}

As the first step to understand the interaction among players of the ISPs, the CPs and end users, a simplified model with one ISP, one CP and large number of users is considered. The price charged by the ISP for the CP is $p^{\mathrm{t}}$ per unit demand. The ISP charge usage-based fee $p^{\mathrm{s}}$ from end users, where $p^{\mathrm{s}}$ is the non-negative price of per unit demand. The CP charges users a non-negative price $p^{c}$ per unit of content within a finite time. Though $p^{c}$ has a different unit as $p^{\mathrm{s}}$, it can be mapped from the price per content into the price per bps. The set of $p^{\mathrm{s}}$ and $p^{\mathrm{c}}$ is denoted as

$$
S_{\mathrm{ISP}}=\left\{p^{\mathrm{s}} \mid p^{\mathrm{s}} \geq 0\right\}
$$

and

$$
S_{\mathrm{CP}}=\left\{p^{\mathrm{c}} \mid p^{\mathrm{c}} \geq 0\right\}
$$

respectively. The users demand is a function of $p^{\mathrm{s}}$ and $p^{\mathrm{c}}$. We define the users generic demand function by $D$ as follows:

$$
D\left(p^{\mathrm{s}}, p^{\mathrm{c}}\right)=\max \left\{0, D_{0}-\alpha p^{\mathrm{s}}-\beta p^{\mathrm{c}}\right\}
$$

where $D_{0}, \alpha$ and $\beta$ are all positive constants. The parameter $D_{0}$ reflects the total potential demand of users. The parameters $\alpha$ and $\beta$ denote the responsiveness of demand to the price (note that in economics, we also call the parameter $\alpha$ and $\beta$ as price elasticity of demand [13]). The meaning of Eq. (3) can be interpreted as this way: when the prices of ISP and CP increase (or decrease), the demand from users decreases (or increases). 
Therefore, the payoff of ISP can be expressed as follows:

$$
\Pi_{\mathrm{ISP}}=\left(p^{\mathrm{s}}+p^{\mathrm{t}}\right) D\left(p^{\mathrm{s}}, p^{\mathrm{c}}\right)
$$

The payoff of $\mathrm{CP}$ can be expressed as follows:

$$
\Pi_{\mathrm{CP}}=\left(p^{\mathrm{c}}-p^{\mathrm{t}}\right) D\left(p^{\mathrm{s}}, p^{\mathrm{c}}\right)
$$

\section{STACKELBERG-BERTRAND MODELLING}

We propose a novel game called Stackelberg-Bertrand game to model the noncooperative behaviours between ISP and CP. At the first stage of the Stackelberg-Bertrand game, the ISP sets price $p^{t}$ for the CP. At the second stage of the game, given the price $p^{\mathrm{t}}$, ISP and CP play a Betrand game by simultaneously setting $p^{\mathrm{s}}$ and $p^{\mathrm{c}}$ for end users respectively. In order to solve the equilibrium price of the game, we adopt the method of backward induction in which the second stage game is solved firstly and then the first stage is solved.

The proposed Stackelberg-Bertrand game modelling is shown in Figure 2.



Figure 2: Stackelberg-Bertrand game modeling.

Now we consider the second stage game firstly. Given the price $p^{\mathrm{t}}$, we formulate the game between ISP and CP in the second stage as a Bertrand (price) competition game. The Bertrand game, $\Gamma$ (Player, Strategy, Payoff), is described as follows:

- Player: The ISP and CP are players of this game.

- Strategy: For the ISP, the strategy is the usage price charged users; and for the $\mathrm{CP}$, the strategy is the content price charged users.

- Payoff: For both the ISP and CP, the payoffs are given in Eq. (4) and Eq. (5), respectively.

Nash equilibrium (NE) is a profile of strategies such that no player in the game can improve its payoff by changing its own strategy unilaterally. In other words, each player is doing the best response to the others. For the NE of the above Bertrand game, we have the following definition.
Definition 1. Given specific $p^{\mathrm{t} *}$, the NE point $\left\{p^{\mathrm{s} *}, p^{\mathrm{c} *}\right\}$ of game $\Gamma$ can be expressed as follows:

$$
\begin{aligned}
& p^{\mathrm{s} *}=\arg \max _{p^{\mathrm{s}} \in S_{\mathrm{ISP}}} \Pi_{\mathrm{ISP}}\left(p^{\mathrm{s}}, p^{\mathrm{c} *}, p^{\mathrm{t} *}\right) \\
& p^{\mathrm{c} *}=\arg \max _{p^{\mathrm{c}} \in S_{\mathrm{CP}}} \Pi_{\mathrm{CP}}\left(p^{\mathrm{s} *}, p^{\mathrm{c}}, p^{\mathrm{t} *}\right)
\end{aligned}
$$

In NE, neither ISP nor $\mathrm{CP}$ can improve its payoff by changing its own price unilaterally. Both ISP's payoff and CP's payoff are maximized at NE point.

Proposition 1. The NE point $\left\{p^{\mathrm{s} *}, p^{\mathrm{c} *}\right\}$ of Bertrand game $\Gamma$ can be expressed as Eq. (8) and Eq. (9)

$$
\begin{aligned}
& p^{\mathrm{s} *}=\frac{D_{0}-(2 \alpha+\beta) p^{\mathrm{t}}}{3 \alpha} \\
& p^{\mathrm{c} *}=\frac{D_{0}+(2 \beta+\alpha) p^{\mathrm{t}}}{3 \beta}
\end{aligned}
$$

Proof. To maximize $\Pi_{\text {ISP }}$, we have the following optimal condition,

$$
\frac{\mathrm{d} \Pi_{\mathrm{ISP}}}{\mathrm{d} p^{\mathrm{s}}}=0
$$

By solving Eq. (10), we have

$$
\begin{aligned}
p^{\mathrm{s}} & =B R_{\operatorname{ISP}}\left(p^{\mathrm{c}}\right) \\
& =\frac{D_{0}-\alpha p^{\mathrm{t}}-\beta p^{\mathrm{c}}}{2 \alpha}
\end{aligned}
$$

The optimal price of ISP is a function of $p^{\mathrm{c}}$ given specific $p^{\mathrm{t}}$, which is defined as function $B R_{\mathrm{ISP}}\left(p^{\mathrm{c}}\right)$. In game theory [5], we call the function $B R_{\mathrm{ISP}}\left(p^{\mathrm{c}}\right)$ as best response function of the player ISP.

Similarly, to maximize $\Pi_{\mathrm{CP}}$ by letting

$$
\frac{\mathrm{d} \Pi_{\mathrm{CP}}}{\mathrm{d} p^{\mathrm{c}}}=0
$$

We can get the best response function of player $\mathrm{CP}$ as follows.

$$
\begin{aligned}
p^{\mathrm{c}} & =B R_{\mathrm{CP}}\left(p^{\mathrm{s}}\right) \\
& =\frac{D_{0}+\beta p^{\mathrm{t}}-\alpha p^{\mathrm{s}}}{2 \beta}
\end{aligned}
$$

Combining Eq. (11) and Eq. (13), we can get the Nash equilibrium point as the following

$$
\begin{aligned}
& p^{\mathrm{s} *}=\frac{D_{0}-(2 \alpha+\beta) p^{\mathrm{t}}}{3 \alpha} \\
& p^{\mathrm{c} *}=\frac{D_{0}+(2 \beta+\alpha) p^{\mathrm{t}}}{3 \beta}
\end{aligned}
$$

Secondly, the ISP sets price $p^{t}$ to maximize his payoff.

Lemma 1. The users' demand in the second stage Bertrand game can be expressed as in Eq. (16)

$$
D\left(p^{\mathrm{s} *}, p^{\mathrm{c} *}\right)=\frac{D_{0}+(\alpha-\beta) p^{\mathrm{t}}}{3}
$$


Proof. By substituting the Eq. (8) and Eq. (9) into Eq. (3), we can get the demand as Eq. (16) at the NE point.

Lemma 1 expresses the demand as a function of $p^{\mathrm{t}}$. By substituting Eq. (8) and Eq. (16) into Eq. (4), we can express the payoff of the ISP as function of $p^{t}$ as the following Eq.(17),

$$
\Pi_{\text {ISP }}=\frac{\left[D_{0}+(\alpha-\beta) p^{\mathrm{t}}\right]^{2}}{9 \alpha}
$$

Similarly, by substituting Eq. (9) and Eq. (16) into Eq. (4), we can express the payoff of the $\mathrm{CP}$ as a function of $p^{\mathrm{t}}$ as the following Eq.(18),

$$
\Pi_{\mathrm{CP}}=\frac{\left[D_{0}+(\alpha-\beta) p^{\mathrm{t}}\right]^{2}}{9 \beta}
$$

We evaluate the effect of $p^{t}$ on the payoff the ISP and the $\mathrm{CP}$ by numeric analysis in section 4 .

\section{NUMERIC ANALYSIS}

Numerical results are presented in this section to show how the price and payoff of ISP and CP evolve when the price charged to CP by ISP changes.

We consider three kinds of demand function:

$$
\begin{aligned}
& D\left(p^{\mathrm{s}}, p^{\mathrm{c}}\right)=100-2 p^{\mathrm{s}}-4 p^{\mathrm{c}} \\
& D\left(p^{\mathrm{s}}, p^{\mathrm{c}}\right)=100-2 p^{\mathrm{s}}-3 p^{\mathrm{c}} \\
& D\left(p^{\mathrm{s}}, p^{\mathrm{c}}\right)=100-4 p^{\mathrm{s}}-2 p^{\mathrm{c}}
\end{aligned}
$$

The NE points of the Bertrand game are shown in Figure 3 and Figure 5 under above three kinds of demand functions. The payoffs of ISP and CP are shown in Figure 4 and Figure 6 under above three kinds of demand functions.

We can see from Figure 3 and Figure 4 that the relative price elasticity of ISP and CP plays an important part in price strategy of ISP and CP. When $\alpha$ equals to 2 and $\beta$ equals to 4 , the ISP gets much higher payoff by setting a relative lower $p^{\mathrm{t}}$. When $\alpha$ equals to 4 and $\beta$ equals to 2 , the ISP tends to set a much higher $p^{t}$ to pursue much more payoff.

We can see from Figure 5 and Figure 6 , when $\alpha$ equals to 2 and $\beta$ equals to 4 , the ISP gets much lower payoff than that when $\alpha$ equals to 2 and $\beta$ equals to 3 . When $\alpha$ equals to 4 and $\beta$ equals to 2 , the prices $p^{\mathrm{s}}$ and $p^{\mathrm{c}}$ are much lower than that when $\alpha$ equals to 2 and $\beta$ equals to 3 .

\section{CONCLUSIONS}

By adopting a generic user demand function, this paper proposed a novel Stackelberg-Bertrand game to study the noncooperative behaviour between ISP and CP, the Nash

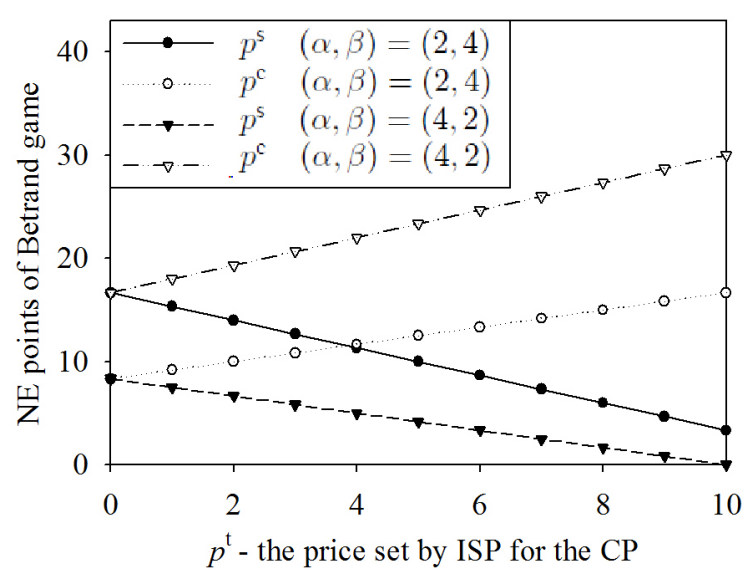

Figure 3: NE point prices of ISP and CP.

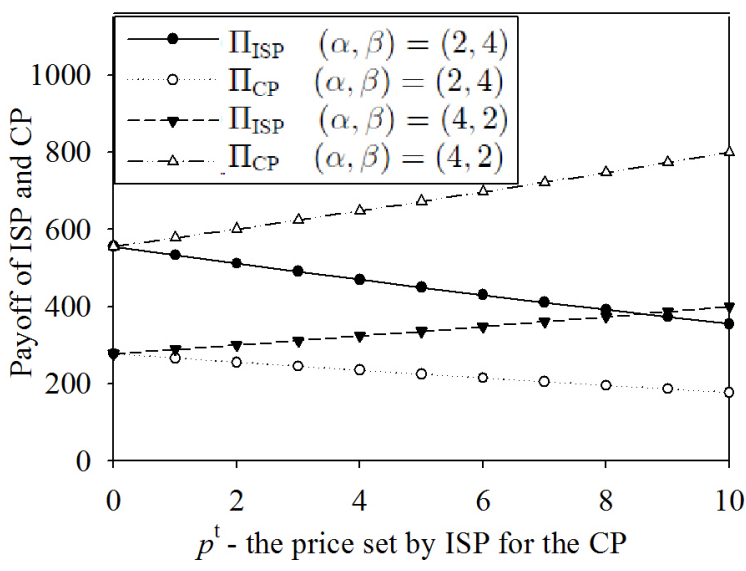

Figure 4: Payoff of ISP and CP.

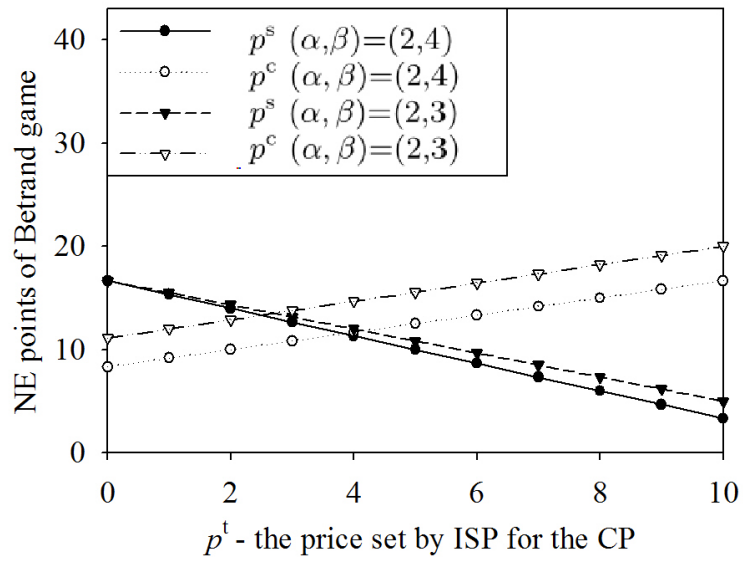

Figure 5: NE point prices of ISP and CP.

equilibrium of the Bertrand game between the ISP and CP 




Figure 6: Payoff of ISP and CP.

has been established and impact of payment charged by ISP for CP has been evaluated.

The numerical results show that the price elasticity of ISP and CP plays an important part on the payoff of the ISP and CP. When price elasticity of ISP is less than that of CP, the ISP gets much higher payoff by setting a relative lower price for CP. When price elasticity of ISP is higher than that of $\mathrm{CP}$, the ISP tends to set a much higher price for $\mathrm{CP}$ to pursue much more payoff.

This paper only considered one ISP, one CP and many users. The case that many ISPs and many CPs should be considered in the future work. In this case, there are competitions among ISPs as well as competitions among CPs. For CP, only the revenue from users' subscription is considered. But many CPs actually generate revenue from advertisement that inserted into contents. The model that incorporate the revenue from advertisement should also be considered in the future work.

\section{REFERENCES}

[1] The Ministry of Internal Affairs and Communictions of Japan, White paper for network neutrality. http://www.soumu.go.jp/joho_tsusin/eidsystem/ program_old07.html, 2007. [Online; accessed 31-January-2015].

[2] E. Altman, A. Legout, and Y. Xu. Network non-neutrality debate: An economic analysis. In Proceedings of 10th International IFIP TC 6 Networking Conference Part II, IFIP '11, pages 9-13, Valencia, Spain, May 2011. Springer Berlin Heidelberg.

[3] S. Boyd and L. Vandenberghe. Convex Optimization. Cambridge University Press, Cambridge, UK, 2004.

[4] F.P.Kelly, A.K.Maulloo, and D. Tan. Rate control for communication networks:shadow prices, proportional fairness and stability. Journal of the Operational Research Society, 49(3):237-252, 1998.

[5] D. Fudenberg and J. Tirole. Game Theory. The MIT Press, Massachusetts, MA, USA, 1991.

[6] P. Hande, M. Chiang, R. Calderbank, and S. Rangan.
Network pricing and rate allocation with content provider participation. In Proceedings of IEEE Conference on Computer Communications, INFOCOM '09, pages 990 - 998, Rio de Janeiro, Brazil, April 2009. IEEE Computer Society.

[7] C. Joe-Wong, S. Ha, and M. Chiang. Sponsoring mobile data: An economic analysis of the impact on users and content providers. In Proceedings of IEEE Conference on Computer Communications, INFOCOM '15, Hong Kong, China, April 2015. IEEE Computer Society.

[8] F. P. Kelly. Charging and rate control for elastic traffic. European Transactions on Telecommunications, 8(1):33-37, 1997.

[9] T. Ma, D. Chiu, J. Lui, V. Misra, and D. Rubenstein Internet economics: The use of shapley value for isp settlement. IEEE/ACM Trans. Netw., 18(3):775-787, 2010.

[10] T. Ma, D. M. Chiu, J. Lui, V. Misra, and D. Rubenstein. On cooperative settlement between content, transit, and eyeball internet service providers. IEEE/ACM Trans. Netw., 19(3):802-815, 2011.

[11] J. Musacchio, J. Walrand, and G. Schwartz. Network non-neutrality debate: An economic analysis. In Conference Record of the Forty-First Asilomar Conference on Signals, Systems and Computers, ACSSC '07, pages 1437-1444, Pacific Grove, CA, USA, November 2007. IEEE Computer Society.

[12] A. Roth. The Shapley value: Essays in honor of Lloyd S. Shapley. Cambridge University Press, Cambridge, UK, 1988.

[13] P. Samuelson and W. Nordhaus. Microeconomics. McGraw-Hill, New York, NY, 2009.

[14] L. S. Shapley. A Value for n-person Games. Princeton University Press, New Jersey, NJ, USA, 1953.

[15] J. Tirole. The Theory of Industrial Organization. The MIT Press, Massachusetts, MA, USA, 1988.

[16] Z.-L. Zhang, P. Nabipay, A. Odlyzko, and R. Guerin. Net neutrality: The technical side of the debate: A white paper. In ACM SIGCOMM Computer Communication Review, volume 37 of $C C R$ ' $0 \%$, pages 49-56, New York, NY, USA, January 2007. ACM. 